\title{
Glottopol
}

Revue de sociolinguistique en ligne

$34 \mid 2020$

Les « langues de France ", 20 ans après

\section{La langue bretonne : une visibilité toute en retenue}

Hervé Le Bihan

\section{OpenEdition}

Journals

Édition électronique

URL : https://journals.openedition.org/glottopol/475

DOI : $10.4000 /$ glottopol.475

ISSN : 1769-7425

Éditeur

Presses universitaires de Rouen et du Havre

Référence électronique

Hervé Le Bihan, «La langue bretonne : une visibilité toute en retenue », Glottopol [En ligne], 34 | 2020, mis en ligne le 01 juillet 2020, consulté le 02 octobre 2021. URL : http://journals.openedition.org/ glottopol/475; DOI : https://doi.org/10.4000/glottopol.475

Glottopol 


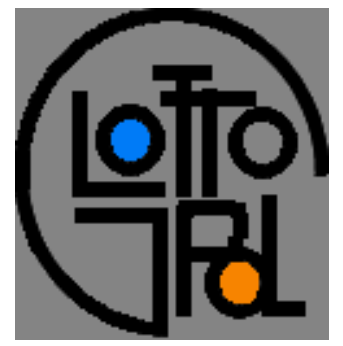

\section{GLOTTOPOL}

Revue de sociolinguistique en ligne $\mathrm{n}^{\circ} 34$ - juillet 2020

Les "langues de France " : 20 ans après

\section{SOMMAIRE}

Hommages à Jean Le Dî

Christian Lagarde : "Langues de France ». Au-delà du symbolique?

Entretien avec Bernard Cerquiglini, par Christian Lagarde.

Entretien avec Paul de Sinety, Délégué général à la langue française et aux langues de France, par Christian Lagarde.

Georg Kremnitz : La problématique initiale de la liste Cerquiglini et ses effets ultérieurs.

Alain Viaut : De "langue régionale » à "langue de France » ou les ombres du territoire.

Wanda Mastor: Le statut constitutionnel des langues régionales en droit comparé. De la reconnaissance à l'indifférence.

Philippe Martel, Marie-Jeanne Verny: Les langues régionales au Parlement, ou l'éternel retour.

Romain Colonna : Les «langues de France »: des langues non-étatiques au pays de l'Étatnation.

Hervé le Bihan : La langue bretonne : une visibilité toute en retenue.

Véronique Bertile : Les langues d'outre-mer : des langues de France? Approche juridique.

Jacques Vernaudon: Les langues polynésiennes et kanak, des "langues de France» en contexte de décolonisation.

Luc Biichlé : Qu'advient-il de l'arabe de France? Mise en perspective sociolinguistique...

Pascal Ottavi : L'épervier, la cage et le passereau.

Marielle Rispail : Le francique lorrain, langue de France? Réflexions et témoignages.

\section{Compte rendu de lecture}

Par Salih Akin : Jean Le Dû \& Yves Le Berre, Métamorphoses. Trente ans de sociolinguistique à Brest (1984-2014), Brest, Centre de Recherche Bretonne, 2019, 302 p.

http://glottopol.univ-rouen.fr 


\title{
LA LANGUE BRETONNE : UNE VISIBILITÉ TOUTE EN RETENUE.
}

\author{
Hervé Le Bihan \\ Université Rennes 2
}

La langue bretonne, aujourd'hui, se trouve à une croisée des chemins, pour reprendre le titre du rapport de 2007 sur l'état de la langue produit par l'Office Public de la Langue Bretonne'. Ce qui était constaté en 2007 est toujours aussi vrai.

À une croisée des chemins, car sa visibilité augmente de plus en plus sur le terrain public, grâce à diverses interventions, mais aussi parce que le principe de subsidiarité qui avait permis, jusqu'ici, de concrétiser divers pans de la demande sociale, dont faire progresser l'usage et la pratique de la langue, se trouve aujourd'hui en passe d'être inopérant... du fait de sa réduction à une peau de chagrin. L'institution régionale, acquise à une consolidation linguistique, ne peut nullement prendre le relais complet par le biais d'une politique linguistique véritable pour une raison bien connue : les assemblées régionales ne peuvent légiférer, elles ne peuvent que dépenser un maigre budget.

Parallèlement les mentalités ont évolué et la vision d'une langue inscrite dans les textes cède la place à une vision bien plus pragmatique, celle de donner des droits aux brittophones ${ }^{2}$.

Il faut rappeler que jusque vers les années 70-80, c'est-à-dire la création des écoles Diwan (1977) puis des écoles publiques bilingues, et enfin des écoles privées catholiques bilingues, la pyramide des âges de la langue collait à celle des vieilles générations qui disparaissaient les unes après les autres, emportant la langue non transmise avec elles. Suite à ces créations, un choc psychologique a été opéré et a mis en lumière le véritable problème de la langue : celui de sa transmission. Deux courants disparitaires ont alors prospéré côte à côte, d'une part celui des militants de longue durée, aussi ceux qui avaient muri ces créations, et qui n'avaient qu'un seul but, celui de relancer la transmission familiale, et d'autre part celui des milieux proches des politiques qui voyaient en l'école la bouée de sauvetage de la transmission de la langue, et aussi une justification simple à l'immobilisme de politique linguistique.

\section{Visibilité et mémoire linguistiques}

C'est au cœur des années 80 que le militantisme radical breton commença à obtenir de sérieuses avancées sur la signalisation. En effet, un groupe nommé Stourm ar Brezhoneg (SAB)

\footnotetext{
${ }^{1}$ http://www.fr.brezhoneg.bzh/46-situation-de-la-langue.htm

${ }^{2}$ Le terme brittophone est un néologisme préférable au mot bretonnant qui est à la fois ambigu - il ne désigne pas que les locuteurs mais aussi ceux «s'occupent » d'affaires bretonnes, culturelles ou non - et péjoratif (voir Le Menn, 1992). Le terme même de francophone est un néologisme récent daté de 1932 d'après le TLFi.
} 
( «le combat/la lutte de la langue bretonne ») ${ }^{3}$, constitué sur le modèle militant de Cymdeithas yr Iaith Gymraeg («la société de la langue galloise »), avait lancé une rude campagne pour obtenir une signalisation en langue bretonne sur les routes bretonnes, qu'elles soient locales, départementales ou nationales. Il s'agissait de détruire les panneaux routiers monolingues par divers moyens, pour forcer les autorités à les remplacer par des panneaux portant les mentions bretonnes des toponymes. Ces diverses campagnes heurteront de plein fouet certaines actions menées bien plus pacifiquement mais bien plus lentes en résultats, comme celles des élus bretonnants constitués en association et présidée par le député-maire puis sénateur-maire socialiste de Cavan, Pierre-Yvon Trémel (1946-2006).

À la suite de négociations entre les militants de Stourm ar Brezhoneg, des élus de tout bord (surtout du Finistère) et les services de l'État, la visibilité de la langue bretonne ne cessera de grandir sur la signalisation routière. Bien entendu il faut voir que mettre les mentions bretonnes des communes tient à la fois de la vision patrimonialiste, bien souvent la seule acceptée par nombre d'élus et les diverses administrations, et de la vision prospective de la langue tenue par les militants. Ce fut un premier terrain d'entente à partir de conceptions différentes, de discours différents sur la langue bretonne ${ }^{4}$.

Mais les choses furent bel et bien lancées : voir la langue écrite de manière «officielle »a provoqué une mutation de la demande sociale. Jusque-là la demande sociale était portée par une poignée de militants, ceux qui avait créé Diwan ${ }^{5}$ en 1977 par exemple, d'organisateurs de cours du soir, etc.

Ainsi outre la place donnée aux toponymes rectifiés en breton, il y a une sorte d'amplitude bien marquée qui voit apparaitre très rapidement les mentions indicatrices ordinaires (centreville, préfecture, parking, etc.). Les échelons locaux et départementaux jouent le jeu de la signalétique bilingue.

La création de Diwan fut un acte primordial dû à une demande sociale structurée, militante. Sans cette structuration, il n'y aurait pas pu avoir de succès de la part d'initiatives individuelles, comme la création de la licence de breton en 1982 ou encore du Capes de breton en 1986. Bien entendu, lorsque nous parlons d'initiative personnelle, nous parlons d'actions individuelles assimilables à du lobbying. Mais sans le relais de la communauté brittophone militante, ces lobbyings n'auraient pu aboutir.

C'est cette amplitude remarquable qui permet cette mutation de la demande sociale à partir des années 90. La visibilité physique de la langue remet dans les esprits la possibilité d'une langue sociétale : ce choc visuel est en rupture avec la vision constamment colportée par divers acteurs politiques ou administratifs (administrations préfectorales, cours de justice, administration rectorale, etc.) selon lesquels «La langue bretonne ne s'écrit pas ». Là aussi il s'agit d'un discours régressif, parfois repris par certains acteurs de langue bretonne ${ }^{6}$, qui bien souvent gomme la mémoire linguistique de la langue bretonne et surtout celle de ses locuteurs, qu'ils soient en filiation directe avec les générations précédentes ou qu'ils soient en insertion linguistique sans histoire linguistique familiale de filiation ${ }^{7}$.

\footnotetext{
${ }^{3}$ Un autre groupe, Skol an Emsav, avait quelques temps auparavant lancé une campagne de bretonnisation des panneaux routiers. Nombre de militants de Skol an Emsav rejoindront Stourm ar Brezhoneg par la suite. Aujourd'hui Skol an Emsav a changé de créneau militant et se consacre essentiellement à la formation longue pour adulte. En ce qui concerne Stourm ar Brezhoneg voir Laquaine-Jakez (2015).

${ }^{4}$ Bien plus tard, le discours d'un ancien président du Conseil Général du Finistère, alors en exercice, confirmera bien cette différence de vision : oui à l'enseignement du breton en option, non à la généralisation d'un système d'enseignement bilingue.

5 Écoles en système d'immersion linguistique. Les autres systèmes, à parité, publics et privés catholiques, apparaissent les années suivantes.

${ }^{6}$ Souvent sous couvert d'une pseudo opposition entre breton populaire et breton littéraire (les termes ne sont pas de nous mais des tenants de cette opposition).

${ }^{7}$ Nous développons ce point plus loin.
} 
C'est l'activation de cette mémoire qui permet de comprendre que la langue bretonne perd constamment sur son propre terrain.

Une fois de plus il faut revenir aux années 1980. Ces années représentent la distance d'une génération suivant celle qui admet la non transmission familiale de la langue comme une règle désormais commune à tous. Car c'est dans les années 60 que les premiers effets de la désaffection de la langue bretonne en tant que langue sociétale généralisée se font sentir. Les événements de 1968 passent par là avec leurs soubresauts et leurs contradictions, dont les contradictions identitaires conjuguées à une conscientisation due aux guerres post-coloniales françaises (en particulier la guerre d'Algérie) ${ }^{8}$. C'est aussi la période du pompidolisme triomphant, clairement opposé aux langues régionales ${ }^{9}$.

Dans les années 80, la mémoire linguistique est encore bien présente. C'est celle qui nourrit la demande sociale. Les jeunes militants, pas tous locuteurs de naissance, loin s'en faut, ont encore la mémoire de cette société où la langue bretonne était majoritaire. C'est aussi dans les années 80 que l'on prend une conscience accrue de la nécessité d'une réaction sociétale à court terme.

Le discours alors oscille entre « sauver la langue » et « reconnaitre les droits des locuteurs » : l'articulation militante n'est pas toujours évidente sur ce point. Le discours officiel, lui, ne varie pas : la langue bretonne doit disparaitre, elle n'est pas nécessaire à l'évolution sociale ou économique de son territoire d'origine.

C'est aussi dans les années 80 que la diaspora bretonne sera à son point culminant en tant que masse de population: on arrive au bout du système d'exode rural, connu aussi bien en région parisienne que dans l'espace des villes bretonnes, que l'on définissait alors comme étant des « villes moyennes $»^{10}$.

Ce n'est donc pas un hasard si, d'une part, un habitant de la région de Guingamp, monolingue $^{11}$, n'eut pas le droit à un interprète devant la cour d'assise, alors qu'il était accusé de meurtre, et si d'autre part, certains militants de $S A B$ eurent, eux, le droit à des interprètes. Mais dans le second cas, les affaires passaient en correctionnelle. Ce n'était pas le même niveau de justice et donc pas le même niveau d'implication d'une décision admettant un interprète ou pas. Tout ceci se passant à la même époque, dans des juridictions géographiquement proches, dans le cours des années 80.

Aujourd'hui, en 2020, la mémoire linguistique est profondément transformée. Dans la majorité des terroirs, la langue n'est plus liée à une structure sociale bien identifiée : c'était d'ailleurs le grand reproche qui lui était fait jusqu'aux années 80 , la langue bretonne était la langue d'une société archaïque et peu valorisante.

Cette même mémoire transformée fait que la revendication sociale et linguistique peut être très éloignée de l'histoire initiale. La langue étant de plus en plus déterritorialisée ${ }^{12}$, elle n'est

\footnotetext{
${ }^{8}$ La non reconnaissance de ces événements comme guerre avant le 10 juin 1999, a produit un effet désastreux.

${ }^{9}$ Nous adoptons ce terme, par pure commodité, même s'il recouvre des réalités bien plus nuancées, étendues. Les langues en question sont toutes territorialisées, voire déterritorialisées. La question des diasporas linguistiques reste à étudier.

${ }^{10}$ Comme Lannion, Vannes, Quimper, etc.

${ }^{11}$ Nous considérons comme monolingue un individu qui ne parle qu'une seule langue, ici le breton, et qui au mieux ne fait que plus ou moins comprendre la langue majoritaire, ici le français.

${ }^{12} \mathrm{La}$ déterritorialisation de la langue bretonne n'est pas un phénomène nouveau. Au moyen âge, les étudiants bretons partaient faire leurs études principalement à Orléans, Chartres, Bordeaux et Paris, où les témoignages de leur présence sont nombreux, y compris les brittophones (la première université bretonne sera ouverte à Nantes en 1460). Cette déterritorialisation a pu être autant éphémère que pérenne, menant à la constitution de diasporas. Voir par exemple un témoignage publié par le quotidien Ouest-France: https://www.ouest-france.fr/pays-de-laloire/le-croisic-44490/quand-la-langue-bretonne-debarquait-le-long-des-quais-2019119.
} 
plus en adéquation avec un territoire « exact $»^{13}$. La langue, ou mieux les locuteurs, ne sont plus attachés à un territoire mais à ce que nous appelons la « communauté ${ }^{14}$.

\section{Organiser les communautés et revernaculariser}

Nous l'avons indiqué, la transmission familiale de la langue bretonne est son point faible depuis de nombreuses décennies. Si l'on compare la situation transmissionnelle du corse et du basque, il est évident que la langue bretonne est en position de faiblesse. Les différentes études publiées au début des années 2000 ne donnaient aucun pourcentage pour la transmission familiale du breton : cette transmission était trop faible pour être quantifiée ${ }^{15}$. Aujourd'hui elle est mécaniquement plus élevée, mais bien en-deçà d'une transmission pérenne.

$\mathrm{La}$ «production » de nouveaux locuteurs passe par deux canaux différents : la transmission et l'apprentissage.

Tout d'abord, la transmission familiale, faible, nous l'avons dit. Cette transmission familiale est tributaire de plusieurs conditions et se base sur plusieurs réalités ${ }^{16}$. On peut catégoriser ainsi : une première catégorie, que l'on peut qualifier $d^{\prime}$ ' " historique », est celle des locuteurs qui ont reçu la langue par transmission familiale et qui continuent à le faire, le plus souvent sur une base militante ${ }^{17}$. Une autre catégorie, qui lui est proche, est celle des néolocuteurs ayant reçu la langue plus par «capillarité » que par transmission des parents. Ce dernier cas a été parfaitement identifié dès les années 60-70: la transmission de la langue est dans ce cas clairement liée à l'environnement linguistique immédiat (un des ascendants directs, les ascendants de la génération précédente, le voisinage, et aussi les activités socio-économiques où la langue était encore bien présente ${ }^{18}$. Un troisième cas se présente : celui de quelques individus, souvent des hommes, nés vers 1965-1975, qui ont reçu la langue souvent dans le milieu familial ou proche du milieu familial. Mais, dans ce dernier cas, leur attitude linguistique est fort variée : certains, sans doute une minorité, s'approprient la langue, en font la langue familiale (avec l'accord actif ou passif du conjoint ou de la conjointe) et réinitialisent la transmission intergénérationnelle ${ }^{19}$. Mais, dans cette dernière catégorie, la grande majorité est

\footnotetext{
${ }^{13}$ Comme la Basse-Bretagne traditionnelle.

${ }^{14}$ Le sens du mot « communauté » varie d'une étude à l'autre. Il nous appartient donc d'en préciser le sens dans le cadre de cet article. Nous entendons par «communauté linguistique » un ensemble de locuteurs qui utilisent le breton dans un but communicatif. Il est important de préciser que tous ces locuteurs sont également francophones, hormis certains enfants en bas âge qui n'ont que le breton comme environnement linguistique, ainsi que quelques personnes très âgées (dont la prise en compte est peu pertinente ici). Cette pratique du breton, qui reste à étudier précisément, peut être un choix militant ou un choix naturel ou de première langue (les deux pouvant se confondre). Il importe également de préciser que cette notion de «communauté linguistique » ne repose pas nécessairement sur une aire territoriale précise. Les réseaux sociaux y jouent un rôle important. Pour une étude ciblée sur Plougastel-Saoulas, voir Catalan, 2019. Cette étude prend appui sur la notion d'habitus, développée par Bourdieu (1980, 2008).

${ }^{15}$ Le rapport de l'Office de la Langue Bretonne d'octobre 2002, p. 27, donne un tableau où la transmission familiale n'est pas quantifiable pour le breton, alors que le corse en est à $12 \%$, et l'alsacien à $34 \%$. Voir aussi Broudic (1999) qui analyse les résultats d'un sondage TMO de 1997 donnant des résultats identiques.

${ }^{16}$ Une partie de ce qui suit repose sur des enquêtes personnelles dans le Trégor littoral. Nous avons commencé un certain nombre de recherches sur un secteur délimité par les villes de Lannion, Tréguier, Penvénan et le secteur de La Roche-Derrien, depuis le printemps 2010. Nous avons eu l'opportunité d'interroger une centaine de personnes. Notre focus s'est porté sur la génération née entre 1958 et c.1980, sans négliger les autres locuteurs plus ou moins âgés. Le bilan détaillé de ces recherches sera intégré dans un ouvrage que nous avons en préparation, ouvrage portant sur la transmission générationnelle de la langue bretonne.

17 Madeg (1986).

18 Voir Office (2002) p. 29.

${ }^{19}$ Legendre (2019). Ce travail est novateur et met bien en vue une réappropriation linguistique peu étudiée jusqu'ici, celle qui réactive la transmission intergénérationnelle.
} 
illettrée dans sa langue et ne l'a pas transmise. Ils reproduisent le schéma des années 50-60, lorsque la transmission familiale s'arrêtait de façon assez générale ${ }^{20}$.

À ce problème de transmission, il faut ajouter un problème récurrent de qualité de la langue : la dernière sous-catégorie ci-dessus fait sans doute les frais d'une connaissance par trop empirique de la langue, sans véritable support (éducatif par exemple, mais aussi environnemental). C'est sans doute ce qui peut expliquer ce renoncement à la transmission familiale aux descendants.

Cette dernière sous-catégorie est peu concernée par le système d'enseignement bilingue : rares sont ceux qui mettent leurs enfants dans des classes bilingues. Contrairement à ce que cette série d'images pourrait donner, cette sous-catégorie reste attachée à la langue. Il ne s'agit pas d'un paradoxe mais plutôt da la traduction d'une certaine impuissance à maitriser un futur linguistique.

Les deux premières sous-catégories ont une attitude différente vis-à-vis de ce système scolaire : si l'environnement linguistique est favorable, il y aura adhésion ; en cas contraire, il peut y avoir un refus de mettre ses enfants dans ces classes, car le milieu linguistique familial peut leur apparaitre largement plus favorable ou efficace. La qualité de l'enseignement est au cœur même du bon fonctionnement du système scolaire bilingue ou immersif.

Ensuite, il existe une catégorie devenue brittophone par l'apprentissage. Il s'agit d'adultes, souvent en reconversion professionnelle, où la langue bretonne peut être intégrée au projet futur.

Il existe en Bretagne plusieurs organismes de formation continue pour adultes qui proposent une récupération linguistique par des stages longs (3, 6, 9 mois). Tous ces organismes ${ }^{21}$ bénéficient d'une aide financière de la Région par l'attribution de bourses ${ }^{22}$.

Le cas de l'enseignement bilingue ou immersif interroge également. Le système scolaire est souvent mis en avant pour donner une solution pérenne à la transmission de la langue bretonne : mais apprentissage et transmission ne recouvrent pas les mêmes concepts. Il faut là aussi examiner comment le système scolaire bilingue s'insère dans le passage intergénérationnel. Chez les politiques, l'enseignement bilingue est souvent l'argument : tout comme les panneaux routiers, l'enseignement est quantifiable, est évaluable. Cela permet de donner une image positive d'une politique linguistique affichée. Cela permet surtout d'adresser un retour au corps électoral : les comptes financiers sont toujours l'occasion de valoriser les actions, même les plus simples, même sans grands effets.

Ainsi, il y a affichage d'une progression du nombre d'enfants scolarisés en filières bilingues : le fossé est grand entre cet affichage et la réalité linguistique attendue. Augmenter le nombre de scolaires en filières bilingues, n'est pas augmenter le nombre de locuteurs ${ }^{23}$. On l'a vu, la transmission intergénérationnelle même, trop faible, ne peut prendre un tel relais, du moins aujourd'hui.

Il faut donc s'interroger sur les effets des différentes actions qui devraient aboutir à ce que nous nommons « revernacularisation $»^{24}$. Cette revernacularisation, liée aux conditions socio-

\footnotetext{
${ }^{20}$ Nous en avons fait des observations à partir d'enquêtes personnelles dans une petite zone du Trégor côtier. Voir note 16.

${ }^{21}$ Skol an Emsav, Roudour, Stumdi, Mervent.

${ }^{22}$ La Région attribue également des bourses aux étudiants qui entrent en formation d'enseignants (MEEF PE ou PLC), quelle que soit la filière choisie. De manière curieuse, certains travaux universitaires ne s'intéressent qu'aux sommes dépensées, mais pas aux retombées socio-économiques... Voir Thomas (2014) en ce qui concerne le monde de l'édition littéraire en langue bretonne.

${ }^{23}$ On peut comparer (?) avec la politique linguistique du Gouvernement gallois (Llywodraeth Cymru) qui se donne comme objectif d'atteindre un million de locuteurs en 2050 : https://www.assembly.wales/laid\%20documents/gen-ld11108/gen-ld11108-w.pdf

${ }^{24}$ Moal (2020).
} 
économiques, à l'environnement transmissionnel ou encore à l'apprentissage lié à un projet (professionnel ou personnel) ne peut prendre appui que sur l'existant.

Revernaculariser, c'est remettre la langue au centre des échanges des communautés, sur le plan social, économique, culturel.

La notion même de communauté est essentielle en l'espèce. Les cadrages traditionnels de la société ont subi de fortes mutations depuis la fin de la seconde guerre mondiale, et ces mutations se sont accélérées une première fois dans les années 60 puis dans les années 2000. Cela a pu être parfois décrit en ruptures. Ce qui nous intéresse ici, ce sont les mutations les plus récentes : état final de l'exode rural, développement socio-économique des villes moyennes, perméabilité entre les modes de vie urbain-néo-urbain et rural-néo-rural, développement d'internet et des réseaux sociaux, entre autres.

Ces différentes mutations ont permis à un réseau de communautés de se développer : par exemple, la demande sociale pour obtenir l'ouverture d'une classe bilingue prend fortement appui sur les réseaux sociaux.

Mais les réseaux réels existent bien et sont plutôt bien implantés : le réseau des Maisons de Pays (Ti ar Vro) ${ }^{25}$. Ces centres couvrent pratiquement tout le territoire investi par les brittophones, qu'ils appartiennent à la catégorie « historique » ou à celle de l'apprentissage aux adultes. Ils permettent de fédérer à la fois vie sociale et demande sociale ${ }^{26}$.

La revernacularisation peut atteindre plusieurs niveaux, suivant la force des communautés. Il ne s'agit plus de les situer en milieu rural ou en milieu urbain, mais de les situer en fonction des liens que ces centres peuvent mettre en œuvre entre tous les locuteurs. Il s'agit également d'une manière de revisiter le principe de subsidiarité, principe essentiel à tout fonctionnement démocratique $^{27}$. Ce n'est donc pas un hasard si les Ti ar Vro prennent appui sur l'échelon communal, cantonal. Par exemple, Ti ar Vro du Trégor ${ }^{28}$ est basé à Cavan, une commune d'un peu plus 1500 habitants.

La revernacularisation est tributaire de la vitalité des communautés. Ces communautés portent la demande sociale et cherchent à la concrétiser : investir le terrain du périscolaire, du préscolaire $^{29}$. Le préscolaire, aujourd'hui, prend une importance primordiale: son développement est lié aux mutations sociétales que représente l'accès à une carrière professionnelle des femmes. Les femmes ne sont plus seulement des mères mais aussi des partenaires socio-économiques, l'éducation des enfants étant bousculée dans ses schémas traditionnels. Jusqu'à bousculer la notion de langue maternelle même ${ }^{30}$.

Il est évident que les communautés arrivent rapidement à un point où la demande sociale stagne, et que seule une politique linguistique avec un support législatif fort peut favoriser la revernacularisation.

\footnotetext{
${ }^{25}$ Il existe au pays de Galles un réseau similaire, mais qui a plus de moyens et surtout plus de compétences (centre d'emplois, système de couverture sociale, périscolaire, etc) : Mentrau'r Iaith.

${ }^{26}$ Bien entendu, un Ti ar Vro ne monte pas de dossier pour obtenir une classe bilingue ou pour ouvrir une crèche, mais il permet aux différents acteurs d'avoir un lieu d'échanges et de rencontre, y compris de façon virtuelle.

${ }^{27}$ Depuis 2007, les différentes législatures françaises ont mis à mal ce principe de subsidiarité en cherchant à légiférer sur à peu près tout. On a en mémoire, notamment, les débats autour du rôle et de l'instrumentalisation de l'histoire. Mais surtout, ce principe permettait aux élus locaux de mettre en place des actions pouvant palier les déficiences de l'État concernant les langues régionales.

${ }^{28}$ Grosso-modo, la partie ouest du département des Côtes-d'Armor. Voir : http://www.tiarvro22.bzh/

${ }^{29}$ Le Pelleter (2017).

${ }^{30}$ Blanchet dans Legendre (2019), note 3 p. 15.
} 


\section{Politique linguistique, institutions, textes législatifs républicains et discours}

Depuis le vote de la loi Deixonne en 1951, dont les décrets d'application ont eu un parcours d'une longévité inégalée, l'arsenal législatif concernant la langue bretonne (et donc les autres langues régionales, puisqu'il s'agissait de cet ensemble) est extrêmement maigre. La circulaire Savary de 1982 reste l'une des avancées majeures concernant les systèmes d'enseignement bilingue $^{31}$. On pourrait sans doute faire un gros travail d'édition en nombreux tomes des propositions de lois concernant les langues régionales qui n'ont pas été discutées par les députés, ou alors rejetées ${ }^{32}$. Il faut surtout noter que la loi Deixonne a été adoptée sous la Quatrième République : la Cinquième a bien montré qu'une telle initiative est vouée à l'échec, même si certains élus semblent penser le contraire ${ }^{33}$.

Les institutions républicaines ne sont pas bloquées, elles sont arc-boutées contre l'idée même de donner une solution législative aux langues régionales. On doit sans doute expliquer certaines avancées notables, entre 1981 et 1986 voire 1989, par le fait que le nouveau pouvoir d'alors s'était fortement appuyé sur les réseaux associatifs acquis aux idées socialistes, et que celui-ci faisait un retour en investissement, et non pas en justice linguistique. Les années dites de « rigueur » survenues, ce même pouvoir avait besoin de réactiver tous ses réseaux, y compris ceux des militants associatifs des langues régionales ${ }^{34}$. Il y avait là un clair rapport de force : concessions en échanges d'adhésion, même partielle, à un vote ${ }^{35}$.

La revendication d'un statut institutionnel pour les langues régionales se confond, en partie, avec la demande de la signature de la Charte européenne des langues régionales ou minoritaires (24 juin 1992) $)^{36}$. Cette charte comporte 98 articles : dans les termes de la charte, l'État signataire doit s'engager sur un minimum de 35 paragraphes ou alinéas de la partie III, dont au moins 3 dans chacun des articles 8 (Enseignement), et 12 (Activités et équipements culturels), 1 dans chacun des articles 9 (Justice), 10 (Autorités administratives et services publics), 11 (Médias), et 13 (Vie économique et sociale). La France en a signé 39 (7 mai 1999) qui devraient s'appliquer à une liste de langues non encore établie (malgré les propositions du rapport d'avril 1999 présenté par Bernard Cerquiglini $)^{37}$. On connait la suite, avec la décision négative de ratification du Conseil Constitutionnel (15 juin 1999). Et il ne s'agit pas ici de revenir sur les péripéties entourant cette adoption ou non de la Charte. Il s'agit simplement de montrer que cette demande s'inscrivait dans un quiproquo encore mal évalué. Entre le moment où l'État, par le biais de ses représentants politiques, avait décidé de signer un certain nombre de points de la Charte, et aujourd'hui, il est bien évident que cette ratification serait pour lors contreproductive pour les langues régionales, au moins pour la langue bretonne. Car la demande sociale a réussi à concrétiser la plupart des points listés par la France pour pouvoir signer le texte. Sans entrer dans les détails, ce qui concerne l'enseignement est déjà en place, les médias également, parfois avant l'existence de la Charte, elle-même. Les collectivités territoriales, dont la Région, ont intégré de nombreux points qui apparaissent dans la partie III de la Charte que la France devrait ratifier ${ }^{38}$. Un point cependant, celui qui concerne la demande sociale des

\footnotetext{
${ }^{31}$ Lestic Kervran (2019).

${ }^{32}$ Comme, par exemple, la proposition de loi Destrade en 1984, qui, si elle avait été votée, aurait donné un avenir institutionnel aux différentes langues régionales.

33 Jung \& Urvoas (2012).

${ }^{34}$ Voir plus haut les avancées obtenues par $S A B$.

${ }^{35}$ Des militants de la langue, minoritaires, ont maintenu ce lien de principe qui, jusqu'ici n'a débouché sur aucune avancée significative.

${ }^{36} \mathrm{https}: / / \mathrm{rm} . c 0 e . i n t / 168007 \mathrm{c} 07 \mathrm{e}$

${ }^{37} \mathrm{https} / /$ www.vie-publique.fr/sites/default/files/rapport/pdf/994000719.pdf

38 Quelques $\quad$ exemples. Pour le Finistère :

https://www.finistere.fr/var/finistere/storage/original/application/f47cea1f45775240319803881438522b.pdf
} 
diasporas semble difficile à mettre en place sans arsenal juridique (comme celui proposé par la Charte).

Si bien qu'une signature aujourd'hui serait d'un gain faible, et surtout elle serait une caution pour les minimalistes de la langue. Cela éviterait bien sûr de produire un texte législatif protégeant les droits des locuteurs, et à long terme, une telle ratification serait un obstacle majeur à un statut législatif réel de la langue. Mais encore une fois, le débat autour de cette Charte s'articule sur l'opposition entre les tenants de la langue patrimoine ${ }^{39}$ et les tenants de la langue d'usage.

Pour notre part, en examinant les différents paramètres (historiques, socio-historiques, socioéconomiques, principalement), il est important de comprendre que la vision législative, si vision il y a, doit se porter sur une loi-cadre ouverte pour toutes les langues régionales, et au-delà, celles comprises dans la liste des "langues de France " ${ }^{40}$. Chaque langue, pour les raisons évoquées, doit pouvoir bénéficier d'un statut qui lui est propre. Ainsi, le breton et le corse peuvent prétendre à un appui institutionnel des régions historiques que sont la Bretagne et la Corse. Pour les autres langues, des adaptations institutionnelles sont nécessaires et primordiales $^{41}$.

Mais le focus a toujours porté sur le statut de la langue, alors, qu'à notre sens, c'est le statut des locuteurs qui importe. Les brittophones ont des devoirs en tant que citoyens français, et ont aussi des droits : mais il y a décalage. L'institution ne reconnait qu'une seule série devoirsdroits, celle du citoyen français qui est censé n'être que francophone. La subsidiarité en droits linguistiques n'existe pas et la confusion entre appartenance citoyenne et pratique linguistique s'en trouve biaisée.

Toute législation doit, pour nous, se porter sur les droits des locuteurs : la revernacularisation en dépend, même si les communautés peuvent se renforcer dans le cadre actuel.

Aucun statut d'officialité n'a sauvé une langue, donc les droits de ses locuteurs mais, sans aucun statut d'officialité, le maintien de la vernacularité ne peut se faire. On en a une preuve assez évidente par le vote continu d'un arsenal linguistique protégeant et promouvant la langue française ${ }^{42}$.

En appui des communautés, certaines actions simples ${ }^{43}$ ont permis de pallier ces déficiences. La création de l'Office public de la langue bretonne, EPCC dépendant de la Région Bretagne, en 1999, a été un acte simple, certes, mais majeur. Pour notre part, nous considérons qu'il est à mettre au même niveau que la création de Diwan en 1977. 1977-1999 : cela correspond à l'espace-temps d'une génération.

Aujourd'hui, l'Office joue un double rôle bien identifié : coordonner et impulser. L'Office coordonne, par exemple, la demande sociale en ouverture de classes bilingues. La campagne

\footnotetext{
pour Lannion-Trégor-Communauté : https:/www.ouest-france.fr/bretagne/lannion-22300/lannion-l-agglo-veutmuscler-sa-pratique-du-breton-6598507

${ }^{39}$ Il faut avoir en mémoire les crispations politiques autour de la loi constitutionnelle $n^{\circ} 92-554$ du 25 juin 1992 qui déclare : « la langue de la République est le français. ».

${ }^{40} \mathrm{Ce}$ qui permettrait d'intégrer les langues de l'émigration, les langues des signes (française comme étrangères), les langues non territorialisées en métropole.

${ }^{41}$ Le cas de l'occitan est emblématique : combien de départements, combien de régions, mais aussi combien de métropoles ? Le cas du basque semble plus simple: ne recouvrant pas l'entité d'un département, c'est la communauté de communes qui joue le rôle de « communauté ».

${ }^{42}$ Il ne s'agit pas ici que de la loi dite Toubon, mais aussi de tous les dispositifs qui imposent la langue française. Par exemple, dans le domaine de l'édition en langue régionale, les éditeurs de revues ou journaux sont tenus de mettre les mentions légales en « langue normale » (sic).

${ }^{43}$ Nous appelons « action simple » une action qui ne demande aucun prérequis législatif particulier, et qui donc peut passer outre les clivages politiques d'une assemblée décisionnelle. Ces actions tiennent en partie du principe de subsidiarité.
} 
Ya d'ar brezhoneg («Oui à la langue bretonne ») ${ }^{44}$ impulse justement nombre d'actions au niveau communal, entrepreneurial, etc.

\section{Conclusion provisoire}

Les locuteurs de langue bretonne souffrent d'un manque de droits linguistiques, alors que leurs devoirs de citoyens leur sont bien notifiés. On s'est interrogé sur la validité d'un statut d'officialité pour la langue bretonne donnant, justement, des droits à ses locuteurs. Une grande partie de ce discours a pu être invalidé par le fait que cette revendication sociale est identifiée tardivement chez les locuteurs: jusqu'aux années 60-70, les locuteurs dits naturels, ne ressentaient pas le besoin de cette revendication; ils étaient dans le processus d'abandon de la langue, car contraints d'acquérir une autre langue, le français. Un quiproquo qui a fait croire que les Bretons ont abandonné leur langue de plein gré : la réalité est plus compliquée. Apprendre le français, bien sûr, car c'était la langue de la promotion sociale, mais sans volonté de délaisser leur langue maternelle ${ }^{45}$. La langue bretonne est passée par le broyage social avec l'aide objective des autorités armées du système d'éducation et de promotion sociale par le français.

Depuis les années 60-70, les choses ont changé et des acteurs de la société bretonne ont cherché à prendre leurs affaires en main, tant sur le plan social qu'économique, culturel et linguistique. La création de Diwan en fait partie, mais aussi celle des filières bilingues dans le public ou le privé, ou encore la création de l'Office public de la langue bretonne. Il faut bien voir que l'Office est une initiative de la société civile reprise et amplifiée par l'institution régionale. Le symbole est fort.

La société civile peut aussi, hors réseau associatif ou impulsion institutionnelle, mettre en place des protocoles permettant de revitaliser la langue bretonne dans le monde économique ${ }^{46}$.

Il est très clair que la revernacularisation passera, in fine, par le réamorçage de la transmission familiale ${ }^{47}$.

Aujourd'hui, la langue bretonne a acquis la visibilité, alors que son nombre de locuteurs a dramatiquement diminué. Mais cette diminution demande à être nuancée : les locuteurs et néolocuteurs n'ont plus le profil des générations qui ont terminé de rompre la transmission intergénérationnelle dans les années 60 . Ce profil, lié aux communautés, est un acteur objectif de la revernacularisation, ou du moins de revitalisation linguistique.

\section{Bibliographie}

Blanchet Philippe, voir Legendre.

Bourdieu Pierre, 2008 [1980], Le sens pratique, Repr., Paris, Éditions de Minuit, coll. « Le sens commun », $474 \mathrm{p}$.

Broudic Fañch, 1999, Qui parle breton aujourd'hui? Qui le parlera demain ?, collection Leoriou bihan, $\mathrm{n}^{\circ}$ 4, Brest, Brud Nevez.

Catalan Marcos, Lena, 2019, Sivi ruz ha digenvez - Oberoù brezhonek e Plougastell-Daoulaz, Master 2 (dir. Herve Le Bihan), Université Rennes 2.

\footnotetext{
${ }^{44}$ Il s'agit d'une campagne incitant les différents acteurs politiques, sociaux, économiques, culturels, à intégrer des actions pour l'emploi de la langue bretonne dans leurs diverses structures : http://www.fr.brezhoneg.bzh/32presentation.htm

${ }^{45}$ Le Coadic (1998), Legendre (2019).

${ }^{46}$ Pichon (2017).

${ }^{47}$ Chantreau (2017).
} 
Chantreau Katell, 2017, Treuzkas ar brezhoneg d'ar vugale? Choazoù ha pleustroù yezhel ar gerent vrezhonek yaouank / Transmettre le breton à ses enfants? Les choix et les pratiques linguistiques des jeunes parents bretonnants, Master 2 (dir. Stefan Moal \& Hugues Pentecouteau), Université Rennes 2. http://www.treuzkas.net/IMG/pdf/2017_memor_master_k.chantreau_treuzkas_bzg_er familhou.pdf

Jung Armand \& Urvoas Jean-Jacques, 2012, Langues et cultures régionales - En finir avec l'exception française, Jean Jaurès Fondation.

Laquaine-Jakez, Tomaz, 2015, Stourm ar Brezhoneg (SAB), Pemzek vloaz a stourm evit ar brezhoneg ofisiel, Master 2 (dir. Herve Le Bihan), Université Rennes 2.

Le Coadic Ronan, 1998, L'identité bretonne, Terre de Brume / PUR.

Legendre Marie-Thé, 2019, Le breton, langue des mères, langue des frères, Yoran embanner. [préface de Philippe Blanchet]

Le Menn Gwennole, 1992, «Les Bretons "tonnants" et le monde des lettrés », in 1491, La Bretagne Terre d'Europe, CRBC Brest, pp. 311-323.

Le Pelleter Stéphanie, 2017, Ar magourioù hag ar magerezed : un dachenn nevez evit dazont ar yezh? 1998-2016, Master 2 (dir. Herve Le Bihan), Université Rennes 2.

Lestic Kervran Morgane, 2019, Deskiñ ha kelenn e brezhoneg en eil derez publik etre al lezenn Deixonne (1951) ha kelc'hlizher Savary (1982), Master 2 (dir. Herve Le Bihan), Université Rennes 2.

Madeg Mikael, 1986, Desevel bugale e brezoneg, Dosier dastumet ha renket, Emgleo Breiz / Ar Skol Vrezoneg.

Moal Stefan, à paraitre en 2020, Institutionnalisation et revernacularisation de la langue bretonne : tuilage plutôt que césure, Actes de la journée d'étude « Entre francisation et démarcation: usages hérités et usages renaissantistes des langues régionales de France », Université de Valenciennes, 14 septembre 2018, Carnets d'Ateliers de Sociolinguistique, LESCLAP, Université de Picardie.

Ofis ar Brezhoneg - Office de la Langue Bretonne, 2002 (octobre), Un avenir pour la langue bretonne? - Rapport sur l'état de la langue bretonne, Rennes.

Pichon Denez, 2017, Les langues minoritaires : une différenciation positive dans le monde de l'économie. Le cas de la langue bretonne, Rennes, Tir.

Thomas Mannaig, 2014, «Une littérature en dépendances. La littérature de langue bretonne 2000-2010 », La Bretagne linguistique, $\mathrm{n}^{\circ}$ 18, Brest, CRBC, pp. 177-203.

\section{Sites consultés (février-mars 2020)}

TLFi (mot francophone) :

http://stella.atilf.fr/Dendien/scripts/tlfiv5/advanced.exe?8;s=3058282575;

Politique linguistique de la Région Bretagne :

https://www.bretagne.bzh/actions/langues-regionales/

Affaire du petit Fañch:

https://fr.wikipedia.org/wiki/Affaire $\mathrm{Fa} \% \mathrm{C} 3 \% \mathrm{~B} 1 \mathrm{ch}$

Sur la Charte européenne :

https://rm.coe.int/168007c07e 


\section{Annexe : L'affaire du tilde sur le n ou l'affaire du prénom Fañch ou comment un signe diacritique pourrait remettre en cause les valeurs de la République française.}

Au cours de l'année 2017, une famille s'est vue refuser l'orthographe Fañch pour le prénom de son petit garçon, au motif que le signe diacritique sur le $\mathbf{n}$ n'était pas dans la liste des signes diacritiques prévus par l'administration pour les prénoms donnés aux nouveaux-nés. L'affaire a fini sur le plan judiciaire, et ses différentes péripéties sont parfaitement expliquées sur le site Wikipedia qui lui est dédié ${ }^{48}$. Nous ne reviendrons pas sur les différents arguments donnés de part et d'autre, si ce n'est pour en ajouter un.

Le ñ apparait depuis le milieu des années 80 sur les panneaux routiers, utilisé pour retranscrire certaines formes bretonnes de toponymes (cf. Skiñvieg : Squiffiec, par exemple, nom d'une commune près de Guingamp) ou encore pour certaines indications secondaires (comme le verbe parkañ «se garer» dans Lec'h-parkañ «parking»). Et ceci, sans que quiconque ne s'en émeuve ${ }^{49}$.

Ce qui nous intéresse ici, c'est ce qui semble être un dénouement institutionnel de l'affaire, accompagné de son paratexte.

La presse s'est faite l'écho du courrier adressé par la Ministre de la Justice, Madame Nicole Belloubet, au Président de l'Assemblée, député du Finistère LREM, Monsieur Richard Ferrand, la semaine du 3 février $2020^{50}$. Ce premier fait est à remarquer : c'est à un député LREM élu en Finistère, mais aussi Président de l'Assemblée Nationale, un proche déclaré du Président de la République, que la Ministre a adressé son courrier, et non au Président PS de la Région Bretagne, Monsieur Loïg Chesnais-Girard, qui s'est fortement impliqué dans la reconnaissance de l'orthographe du prénom Fañch depuis le début de l'affaire. Le message est clair, c'est une affaire reprise en main à son profit par le parti du pouvoir en place, trois ans après le début du processus judiciaire.

Dans ce courrier, il est annoncé un décret qui devrait être opérationnel avant l'été, autorisant l'utilisation du fameux $\tilde{\mathbf{n}}$ dans les prénoms « d'origine régionale ». Mais l'arrivée de ce décret avant l'été signifie bien entendu qu'il arrivera après les élections municipales de mars 2020. Il s'agit ici d'un effet d'annonce, assez grossier tout de même. Les partenaires de la demande sociale ne s'y sont pas trompés ${ }^{51}$.

Ce qui nous intéresse véritablement, c'est le paratexte accompagnant cette annonce ${ }^{52}$. En effet, Madame Belloubet indique dans son courrier que l'utilisation du ñ ne peut se faire que si ceci «n'affecte pas le principe énoncé à l'article 2 de la Constitution selon lequel la langue de la République est le français ». Dans ce même courrier, Madame Belloubet explique que cette acceptation ne pourra se faire qu'après étude d'impact pour qu'un tel prénom puisse être reconnu par tous les systèmes informatiques des administrations: une étude sera donc diligentée pour bien recenser toutes les adaptations nécessaires.

Tout ceci amène à plusieurs commentaires : tout d'abord, à l'orée de nouvelles élections, surtout locales, la langue bretonne fait toujours l'objet d'enjeux qui relèvent plus de l'arithmétique des voix obtenues que d'un vrai souci de planification linguistique. Ensuite, la référence à l'article 2 de la Constitution annonce un recentrage de la part du pouvoir central arc-bouté contre tout droit linguistique autre que celui accordé aux francophones. Enfin, le fait

\footnotetext{
${ }^{48} \mathrm{https} / / /$ fr.wikipedia.org/wiki/Affaire $\mathrm{Fa} \% \mathrm{C} 3 \% \mathrm{~B} 1 \mathrm{ch}$

${ }^{49} \mathrm{Il}$ est important de préciser que ces mentions étaient portées sur des panneaux mis en place par les communes et les départements, l'État ne sentant pas concerné.

${ }^{50}$ Ouest-France du mercredi 5 février 2020, page Bretagne. Article signé Didier Gourin et Flora Chauveau.

${ }^{51}$ L'article de Ouest-France se fait l'écho de la grande prudence du président de Skoazell Vreizh, association qui a soutenu la demande la famille.

${ }^{52}$ Toujours cité d'après l'article de Ouest-France.
} 
d'annoncer une étude d'impact d'un tel décret relèverait de la plaisanterie dans d'autres circonstances : ainsi les services de l'État ignoreraient que pour obtenir $\tilde{\boldsymbol{n}}$ sur un clavier d'ordinateur, il suffit de faire alt +164 , qu'il y a même possibilité de créer un raccourci ? Le cout en formation des personnels serait-il en cause?

Plus sérieusement, si l'on peut dire, il s'agit pour la Ministre, ou celui qui a rédigé le texte pour elle, de mettre une distance citoyenne entre revendication linguistique marginale ${ }^{53}$, paternalisme politique ${ }^{54}$ et le sérieux qu'exigent les principes républicains, qui, eux, sont intangibles ${ }^{55}$.

Il restera cependant un point qu'il faudra expliciter : quel aura été le cout financier réel de la judiciarisation volontaire de cette affaire par le politique, non seulement du côté des parents du petit Fañch et des associations qui les ont accompagnés mais aussi du côté de l'institution, qui jusqu'au bout, a préféré laisser la situation pourrir ${ }^{56}$ ? Les citoyens, en Bretagne comme ailleurs, ont le droit de savoir quel aura été le cout réel de la judiciarisation de cette affaire. La transparence, sur ce point, alimente la confiance entre élus, pouvoir central et citoyens en demande sociale sur le long terme : c'est seulement à ce prix que la démocratie peut fonctionner, faute de statut juridique des locuteurs.

\footnotetext{
${ }^{53}$ Que représente ce phénomène Fañch à l'échelle de la République ? Il y a là un retour aux idéaux d'un jacobinisme triomphant.

${ }^{54}$ Car enfin, il est assez inespéré qu'un parti politique en place puisse prendre en charge une telle demande marginale...

${ }^{55} \mathrm{La}$ Constitution est là pour venir en appui, et en seule référence. La Constitution française n'étant pas inclusive, on sait très bien ce que cela signifie : seuls la langue française et les droits de ses locuteurs sont reconnus, le reste ne peut être, au mieux, que marginal.

${ }^{56}$ Sur le plan strictement juridique, les tribunaux ont fait leur travail. C'était aux politiques de régler ce problème de signe diacritique au plus vite.
} 


\section{GLOTTOPOL}

Revue de sociolinguistique en ligne

Comité de rédaction : Michaël Abecassis, Salih Akin, Sophie Babault, Claude Caitucoli, Véronique Castellotti, Régine Delamotte, Robert Fournier, Stéphanie Galligani, Emmanuelle Huver, Normand Labrie, Foued Laroussi, Benoit Leblanc, Fabienne Leconte, Gudrun Ledegen, Danièle Moore, Clara Mortamet, Alioune Ndao, Isabelle Pierozak, Gisèle Prignitz.

Rédactrice en chef : Clara Mortamet.

Comité scientifique : Claudine Bavoux, Michel Beniamino, Jacqueline Billiez, Philippe Blanchet, Pierre Bouchard, Ahmed Boukous, Pierre Dumont, Jean-Michel Eloy, Françoise Gadet, Monica Heller, Caroline Juilliard, Jean-Marie Klinkenberg, Jean Le Dû (†), Marinette Matthey, Jacques Maurais, Marie-Louise Moreau, Robert Nicolaï, Didier de Robillard, Paul Siblot, Claude Truchot, Daniel Véronique.

\section{Comité de lecture pour ce numéro :}

Salih Akin, Carmen Alén Garabato, Sophie Babault, Philippe Blanchet, Henri Boyer, Véronique Castellotti, Marisa Cavalli, Jean-François De Pietro, Didier de Robillard, Alain Di Meglio, Ksenija Djordjevic, Jean Michel Eloy, Pascale Erahrt, Véronique Fillol, Monica Heller, Robert Fournier, Normand Labrie, Hervé Lieutard, Jean Le Dû (†), Marinette Matthey.

http://glottopol.univ-rouen.fr

ISSN : 1769-7425 